\title{
Experiment Databases
}

\author{
Joaquin Vanschoren and Hendrik Blockeel
}

\begin{abstract}
Inductive databases are usually viewed as databases that contain models, next to data, such that mining the data essentially boils down to querying them for models. This unifies data mining with database querying. In his chapter, we consider experiment databases. Experiment databases are similar to regular inductive databases, but operate at a higher level of abstraction. They store not necessarily the models themselves, but properties of those models, and of the learners and datasets used to construct them. Querying them will reveal information about the behavior of data mining algorithms and the models they result in, when run on particular datasets. Thus, experiment databases unify meta-learning (learning about data mining systems) with database querying. In this chapter we discuss the advantages that experiment databases can offer in terms of openness of research, collaboration, reproducibility of experiments, thoroughness of analysis of experimental results, and correctness of their interpretation. We also describe the (principled) design of a pilot experiment database, and illustrate its use.
\end{abstract}

Joaquin Vanschoren

Department of Computer Science, Katholieke Univeristeit Leuven, Leuven, Belgium e-mail: joaquin.vanschoren@cs.kuleuven.be

Hendrik Blockeel

Department of Computer Science, Katholieke Univeristeit Leuven, Leuven, Belgium LIACS, Universiteit Leiden, The Netherlands

e-mail: hendrik.blockeel@cs.kuleuven.be 



\title{
Experiment Databases
}

\author{
Joaquin Vanschoren and Hendrik Blockeel
}

\section{Introduction}

"Study the past", Confucius said, "if you would divine the future". This applies to machine learning and data mining as well: when developing new machine learning algorithms, we wish to know which techniques have been successful (or not) on certain problems in the past, and when analyzing new datasets, we assess the potential of certain machine learning algorithms, parameter settings and preprocessing steps based on prior experience with similar problems.

Since machine learning algorithms are typically heuristic in nature, much of this information is extracted from experiments. Much like in many other empirical sciences, we collect empirical evidence of the behavior of machine learning algorithms by observing their performance on different datasets. If we have a hypothesis about how algorithms will perform under certain conditions, we test this by running controlled experiments, hopefully discovering empirical laws that contribute to a better understanding of learning approaches. Additionally, exploratory studies also probe many algorithms to study their behavior or to assess their utility on new datasets.

As such, all around the globe, thousands of machine learning experiments are being executed on a daily basis, generating a constant stream of empirical information on machine learning techniques. Unfortunately, most of these experiments are interpreted with a single focus of interest, described only concisely in papers and discarded afterwards, while they probably have many

Joaquin Vanschoren

Department of Computer Science, Katholieke Univeristeit Leuven, Leuven, Belgium e-mail: joaquin.vanschoren@cs.kuleuven.be

Hendrik Blockeel

Department of Computer Science, Katholieke Univeristeit Leuven, Leuven, Belgium LIACS, Universiteit Leiden, The Netherlands

e-mail: hendrik.blockeel@cs.kuleuven.be 
uses beyond their original intent. If we properly store and organize these results, they can be immediately reused by other researchers and accelerate future research. But in order to make this possible, we need a system that can store descriptions of data mining run, including the learners and datasets used, and the models produced.

In this chapter, we present experiment databases (ExpDBs): databases designed to collect all necessary details of machine learning experiments, and to intelligently organize them in online repositories to enable fast and thorough analysis of a myriad of collected results. They engender a much more dynamic, collaborative approach to experimentation, in which experiments can be freely shared, linked together, and immediately reused by researchers all over the world, simply by querying them. As we shall see, the use of such public repositories is common practice in many other scientific disciplines, and by developing similar repositories for machine learning, we similarly aim to create an "open scientific culture where as much information as possible is moved out of people's heads and labs, onto the network and into tools that can help us structure and filter the information" [26].

ExpDBs thus constitute an additional, queriable source of empirical metadata, generated by many different researchers. They are a kind of inductive databases in that they store models which can be queried afterwards; however, they differ from regular inductive databases in a number of ways.

First, an inductive database (IDB) stores a single dataset, together with models that may have been produced from that dataset by running inductive queries, and with properties of those models. An experiment database (ExpDB), on the other hand, stores multiple datasets, multiple learners, and multiple models resulting from running those learners on those datasets.

Second, rather than storing the datasets, learners, and models themselves, an ExpDB may in practice store only descriptions (in terms of predefined properties) of them. In a regular IDB, this would not make sense, as the model itself is what the user is interested in.

Finally, in an IDB, one typically queries the data, or the set of models stored in the database (as in the virtual mining views approach, see Chapter ??), to get a model as a result. In an ExpDB, one typically queries the datasets, models, and experimental results in order to find possible relationships between their properties.

In the following sections, we discuss the main benefits of experiment databases in Sect. 2 and present related work in other scientific disciplines in Sect. 3. Next, we provide a high-level overview of their design in Sect. 4. Finally, we illustrate their use in Sect. 5 by querying an existing experiment database to answer various interesting questions about machine learning algorithms and to verify a number of recent studies.

This chapter is based on prior work on experiment databases $[6,40,42,43]$. 


\section{Motivation}

Thousands of machine learning research papers contain extensive experimental evaluations of learning algorithms. However, it is not always straightforward to interpret these published results and use them as stepping stones for further research: they often lack the details needed to reproduce or reuse them, and it is often difficult to see how generally valid they are.

\subsection{Reproducibility and Reuse}

Indeed, while much care and effort goes into machine learning studies, they are usually conducted with a single focus of interest and summarize the empirical results accordingly. The individual experiments are usually not made publicly available, thus making it impossible to reuse them for further or broader investigation. Moreover, because of space restrictions imposed on publications, it is often practically infeasible to publish all details of the experimental setup, making it, in turn, very hard for other researchers to reproduce the experiments and verify if the results are interpreted correctly. This lack of reproducibility has been warned against repeatedly [21, 34, 29, 17], and some conferences have started to require that all submitted research be fully reproducible [24], adding notices to the ensuing publications stating whether or not the results could be verified.

\subsection{Generalizability and Interpretation}

A second issue is that of generalizability: in order to ensure that results are generally valid, the empirical evaluation must cover many different conditions such as various parameter settings and various kinds of datasets, e.g., differing in size, skewness, noisiness or with or without being preprocessed with basic techniques such as feature selection. Unfortunately, many studies limit themselves to algorithm benchmarking, often exploring only a small set of different conditions. It has long been recognized that such studies are in fact only 'case studies' [1], and should be interpreted with caution.

A number of studies have illustrated that sometimes, overly general conclusions can be drawn. In time series analysis research, for instance, it has been shown that many studies were biased toward the datasets being used, leading to contradictory results [21]. Furthermore, Perlich et al. [30] describe how the relative performance of logistic regression and decision trees depends strongly on the size of dataset samples, which is often not taken into account. Finally, it has been shown that the relative performance of lazy learning and rule induction is easily dominated by the effects of parameter optimization, data 
sampling and feature selection [19]. These studies underline that there are good reasons to thoroughly explore different conditions, or at least to clearly state under which conditions certain conclusions may or may not hold.

\subsection{Experiment Databases}

The idea of (inductive) databases that log and organize all the details of one's machine learning experiments, providing a full and fair account of conducted research, was first proposed by one us (Blockeel) [5] as an elegant way to remedy the low reproducibility and generalizability of many machine learning experiments. Still, this work did not present details on how to construct such a database.

Blockeel and Vanschoren [6] provided the first implementation of an experiment database for supervised classification, and further work details how to query this database to gain insight into the performance of learning algorithms [39, 43].

\subsubsection{Collaborative Experimentation}

However, given the amount of effort invested in empirical assessment, and the potential value of machine learning results beyond the summarized descriptions found in most papers, it would be even more useful to employ such databases to create searchable, community-wide repositories, complete with tools to automatically publish experimental results online. Such repositories would be a tremendously valuable source of unambiguous information on all known algorithms for further investigation, verification and comparison.

It engenders a more dynamic, collaborative form of experimentation, in which as many experiments as possible are reused from previous studies, and in return, any additional experiments are again shared with the community [40]. The experiment databases discussed in this chapter allow exactly this: they offer a formal experiment description language (see Sect. 4) to import large numbers of experiments directly from data mining tools, performed by many different researchers, and make them immediately available to everyone. They can be set up for personal use, to share results within a lab and to create open, community-wide repositories.

\subsubsection{Automatic Organization}

Most importantly, they also make it easy to reuse all stored experiments by automatically organizing them. Every new experiment is broken down to its components (such as the algorithm, parameter settings and dataset used), 
and its results are related to the exact configuration of those components. It then only takes a query (e.g in SQL) to ask for all results under specific conditions. For instance, requesting the parameter settings of an algorithm and its performance results allows to track the general effect of each parameter. Additionally requesting the dataset size allows to highlight what influence that may have on those parameters. As will be illustrated in Sect. 5, such queries allow to quickly peruse the results under different conditions, enabling fast and thorough analysis of large numbers of collected results. The expressiveness of database query languages warrants that many kinds of hypothesis can be tested by writing only one or perhaps a few queries, and the returned results can be interpreted unambiguously, as all conditions under which they are valid are stated in the query itself.

As such, instead of setting up new experiments for each question one may be interested in, often a laborious procedure involving the manual collection of datasets and algorithms and the manual organization of results, one could simply write a query to retrieve the results of hundreds of algorithms on perhaps thousands of datasets, thus obtaining much more detailed results in a matter of seconds.

\subsubsection{Meta-learning}

Experiment databases also serve as a great platform for meta-learning studies $[38,41]$, i.e. to search for useful patterns in algorithm behavior. To this end, it is helpful to link the empirical results to known properties of datasets [25, 31], as well as properties of algorithms, such as the type of model used, or whether they produce high bias or variance error [20]. As such, all empirical results, past and present, are immediately linked to all known theoretical properties of algorithms and datasets, providing new grounds for deeper analysis.

Previous meta-learning projects, especially the StatLog [25] and METAL [8] projects, also collected large numbers of machine learning experiments with the goal of using this meta-data to discover patterns in learning behavior, but these repositories were not developed to ensure reproducibility, were not open to new results, nor facilitated thorough querying.

\subsection{4 e-Sciences}

As will be discussed in Sect. 3, many scientific fields have developed online infrastructures to share and combine empirical results from all over the world, thus enabling ever larger studies and speeding up research. In the resulting deluge of combined experimental results, machine learning techniques have proven very successful, discovering useful patterns and speeding up scientific progress. Still, in an apparent contradiction, machine learning experiments themselves are currently not being documented and organized well enough to 
engender the same automatic discovery of insightful patterns that may speed up the design of better algorithms or the selection of algorithms to analyze new collections of data. We aim to solve this contradiction.

\subsection{Overview of Benefits}

We can summarize the benefits of sharing machine learning experiments and storing them in public databases as follows:

Reproducibility The database stores all details of the experimental setup, thus attaining the scientific goal of truly reproducible research.

Reference All experiments, including algorithms and datasets, are automatically organized in one resource, creating a useful 'map' of all known approaches, their properties, and results on how well they fared on previous problems. This also includes negative results, which usually do not get published in the literature. As such, we get a detailed overview of how algorithms from many studies perform relative to one another, and many aspects of learning behavior, that may only be known to some experts, can be instantly explored by writing a query.

Visibility It adds visibility to (better) algorithms that the user may not have been aware of.

Reuse It saves time and energy, as previous experiments can be readily reused. Especially when benchmarking new algorithms on commonly used datasets, there is no need to run older algorithms over and over again, as their evaluations are likely to be available online. This would also improve the quality of many algorithm comparisons, because the original authors probably know best how to tune their algorithms, and because one can also easily take the stored dataset properties into account to find out how they affect the relative performance of algorithms.

Larger studies It enables larger and more generalizable studies. Studies covering many algorithms, parameter settings and datasets are hugely expensive to run, but could become much more feasible if a large portion of the necessary experiments are available online. Even if many experiments are missing, one can use the existing experiments to get a first idea, and run additional experiments to fill in the blanks. And even when all the experiments have yet to be run, the automatic storage and organization of experimental results markedly simplify conducting such large scale experimentation and thorough analysis thereof.

Integration The formalized descriptions of experiments also allow the integration of such databases in data mining tools, for instance, to automatically log and share every experiment in a study or to reuse past experiments to speed up the analysis of new problems. 


\section{Related Work}

The idea of sharing empirical results is certainly not new: it is an intrinsic aspect of many sciences, especially e-Sciences: computationally intensive sciences, which use the internet as a global, user-driven collaborative workspace.

\section{1 e-Sciences}

In all these scientific fields, both the need for reproducibility and the recognition of the potential value of empirical results beyond the summarized descriptions found in most papers, has led to the creation of online, public infrastructures for experiment exchange. Although these infrastructures have evolved somewhat differently in each field, they do share the same three components:

A formal representation language To enable a free exchange of experimental data, a standard and formal representation language needs to be agreed upon. Such a language may also contain guidelines about the information necessary to ensure reproducibility.

Ontologies Defining a coherent and unambiguous description language is not straightforward. It requires a careful analysis of the concepts used within a domain and their relationships. This is formally represented in ontologies [12]: machine manipulable models of a domain providing a controlled vocabulary, clearly describing the interpretation of each concept.

A searchable repository To reuse experimental data, we need to locate it first. Experiment repositories therefore still need to organize all data to make it easily retrievable.

\section{Bioinformatics}

Expression levels of thousands of genes, recorded to pinpoint their functions, are collected through high-throughput screening experiments called DNAmicroarrays. To allow verification and reuse of the obtained data in further studies, microarray databases [35] were created to collect all such experiments. Experiment submission is even a condition for publication in several journals [4]. To support the sharing of these results, a set of guidelines was drawn up regarding the required Minimal Information About a Microarray Experiment (MIAME [9]). Moreover, a MicroArray Gene Expression Markup Language (MAGE-ML) was conceived so that data could be exchanged uniformly, and an ontology (MAGE-MO) was designed [35] to provide a controlled core vocabulary, in addition to more specific ontologies, such as the Gene Ontology [2]. Their success has instigated similar approaches in related fields, such as proteomics [44] and mass spectrometry data analysis. One remaining draw- 
back is that experiment description is still partially performed manually. Still, some projects are automating the process further. The Robot Scientist [23] stores all experiments automatically, including all physical aspects of their execution and the hypotheses under study. It has autonomously made several novel scientific discoveries.

\section{Astronomy}

A similar evolution has taken place in the field of astronomy. Astronomical observations from telescopes all over the world are collected in so-called Virtual Observatories [36]. This provides astronomers with an unprecedented catalog - a World-Wide Telescope - to study the evolving universe. An extensive list of different protocols supports the automatic sharing of observations, such as XML formats for tabular information (VOTable) [27] and astronomical image data (FITS, including meta-data on how the image was produced), as well an Astronomical Data Query Language (ADQL) [45] and informal ontologies [13]. The data is stored in databases all over the world and is queried for by a variety of portals [32], now seen as indispensable to analyze the constant flood of data.

\section{Physics}

Various subfields of physics also share their experimental results in common repositories. Low-energy nuclear reaction data can be expressed using the Evaluated Nuclear Data File (ENDF) format and collected into searchable ENDF libraries. ${ }^{1}$ In high-energy particle physics, the HEPDATA ${ }^{2}$ website scans the literature and downloads the experimental details directly from the machines performing the experiments. Finally, XML-formats and databases have been proposed for high-energy nuclear physics as well [10].

\subsection{Extension to Machine Learning}

We will use the same three components to develop a similar infrastructure for the exchange of machine learning experiments. While different kinds of machine learning experiments exist, we can similarly express their structure and vocabulary to describe, share and organize them in a uniform fashion.

Moreover, experiments in machine learning should be much easier to manipulate. First, compared to the in vitro experiments in bioinformatics, the exchange of the in silico experiments in machine learning can be automated completely. Indeed, a great deal of experimentation is performed through

${ }^{1}$ http://www.nndc.bnl.gov/exfor/endf00.jsp

2 http://durpdg.dur.ac.uk/hepdata/ 


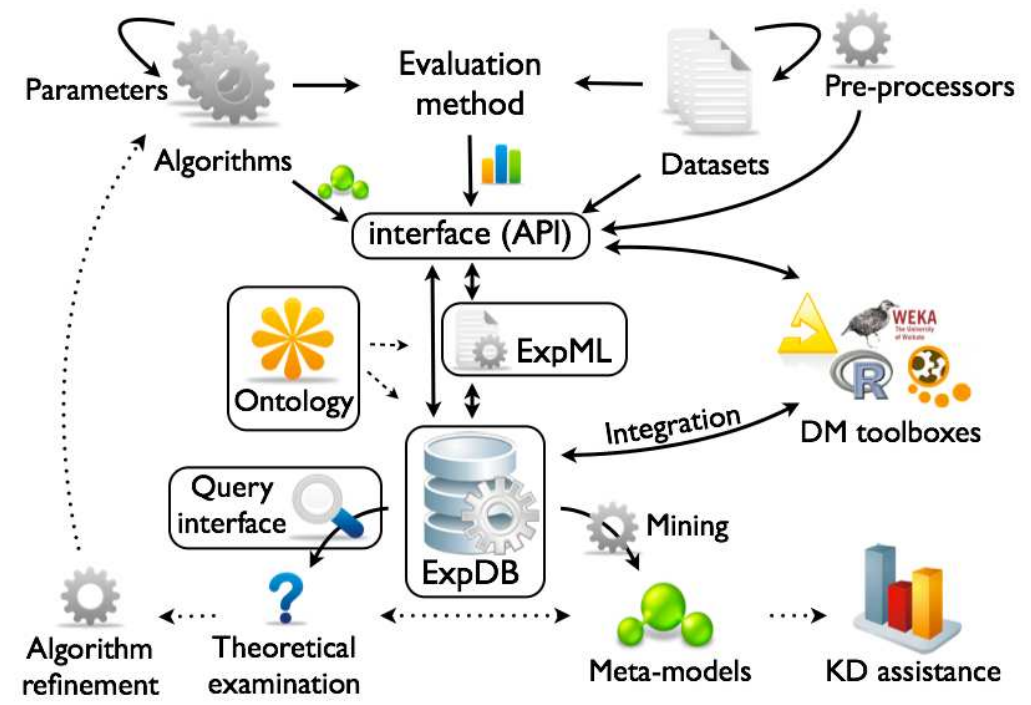

Fig. 1 An online infrastructure for experiment exchange.

data mining workbenches and smaller software tools. As such, experiments could be exported at the click of a button.

Second, in contrast to scientific equipment, we can store datasets and algorithms into the database as well, cross-linked with existing repositories for datasets [3] and machine learning algorithms [34]. As such, all information necessary to reproduce the stored experiments can be found easily.

\section{A Pilot Experiment Database}

In this section, we provide a high-level outline of how we designed our current experiment database, which, although built to extend easily to other tasks, is focused on supervised classification. A detailed discussion is outside the scope of this chapter: we will only highlight the most important aspects of its design and how it can be used in data mining research. All further details, including detailed design guidelines, database models, ontologies and XML definitions, can be found on the ExpDB website: http://expdb.cs.kuleuven.be 


\subsection{Conceptual Framework}

An overview of how experiment databases are used in practice is shown in Fig. 1. The five boxed components include the three components also used in e-Sciences: an ontology of domain concepts involved in running data mining experiments, a formal experiment description language (ExpML) and an experiment database to store and organize all experiments (ExpDB). In addition, two interfaces are defined: an application programming interface (API) to automatically export experiments from data mining software tools, and a query interface to browse the results of all stored experiments. Each is briefly discussed below.

\subsubsection{Software Interface}

First, to facilitate the automatic exchange of data mining experiments, an application programming interface (API) is provided that builds uniform, manipulable experiment instances (java objects) out of all necessary details and exports them as descriptions in ExpML language or directly stores them in a database. The top of Fig. 1 shows some of the inputs. One can describe new algorithms, datasets, evaluation metrics and so on, and in turn use them in the description of new experiments. New elements are described by (among others) name, version, download url and a list of predefined properties, e.g. the number of examples or the skewness of the class attribute in datasets or the type of model used by learning algorithms. The API can also calculate dataset properties for new datasets. Additionally, source code, executable versions of the algorithms or entire datasets can also be stored, although in some situations this may not always be feasible. Finally, the results of the algorithm evaluation and the produced models (or only their predictions) can be described as well.

Software agents such as data mining workbenches (shown on the right hand side in Fig. 1) or custom algorithm implementations can then call methods from the API to create new experiment instances, add the used algorithms, parameters, and all other details as well as the results, and then stream the completed experiments to online ExpDBs to be stored. A multi-tier approach can also be used: a personal database can collect preliminary experiments, after which a subset can be forwarded to lab-wide or community-wide databases.

The ExpDB website currently offers a Java API, including working examples to illustrate its use. It also links to the WEKA platform, allowing the execution and automatic storage of experiments on WEKA algorithms. Further extensions to other platforms, such as KNIME and Rapidminer are also planned. 
This approach is quite different from other, more recent proposals for experiment databases, such as MLComp. ${ }^{3}$ They require algorithms to be uploaded into the system, and scripts to be written that interface with the algorithm execution system.

\subsubsection{The Exposé Ontology}

The vocabulary and structure of the ExpML files and database model is provided by an ontology of data mining experimentation, called Exposé. It provides a formal domain model that can be adapted and extended on a conceptual level, thus fostering collaboration between many researchers. Moreover, any conceptual extensions to the domain model can be translated consistently into updated or new ExpML definitions and database models, thus keeping them up to date with recent developments.

Exposé is built using concepts from several other data mining ontologies. First, OntoDM [28] is a general ontology for data mining which tries to relate various data mining subfields. It provides the top-level classes for Exposé, which also facilitates the extension of Exposé to other subfields covered by OntoDM. Second, EXPO [33] models scientific experiments in general, and provides the top-level classes for the parts involving experimental designs and setups. Finally, DMOP [16] models the internal structure of learning algorithms, providing detailed concepts for general algorithm definitions. Exposé unites these three ontologies and adds many more concepts regarding specific types of experiments, evaluation techniques, evaluation metrics, learning algorithms and their specific configurations in experiments. In future work, we also wish to extend it to cover preprocessing techniques in more depth, for instance using the KD ontology [46] and DMWF ontology [22], which model the wider KD process. The full OWL-DL ${ }^{4}$ description can be found online.

Exposé defines various kinds of experiments, such as 'learner evaluations', which apply a learning algorithm with fixed parameter settings on a static dataset, and evaluate it using a specific performance estimation method (e.g., 10 -fold cross validation) and a range of evaluation metrics (e.g., predictive accuracy). As shown at the top of Fig. 2, experiments are described as workflows, with datasets as inputs and evaluations or models as outputs, and can contain sub-workflows of preprocessing techniques. Algorithms can also be workflows, with participants (components) such as kernels, distance functions or base-learners fulfilling a certain role. The top of Fig. 3 clarifies the structure of workflows: they can have several inputs and outputs, and consist of participants (operators), which in turn can also have multiple in- and outputs. Exposé also differentiates between general algorithms (e.g., 'decision trees'), versioned implementations (e.g., weka.J48) and applications (weka.J48 with

3 http://mlcomp.org

${ }^{4}$ http://www.w3.org/TR/owl-guide/ 
Table 1 Translating ontological properties to XML syntax.

\begin{tabular}{l|l} 
Ontological property & XML syntax \\
\hline has-part, pas-participant & target: subelement of source \\
has-description & (required) attribute \\
has-quality & subelement called property \\
is-concretization-of & implementation_of attribute \\
has-component & target: subelement of source with role attribute \\
has-specified-input & input given id, referenced in input_data attribute \\
has-specified-output & source given id, referenced in output_of attribute
\end{tabular}

fixed parameters). Finally, the context of sets of experiments can also be described, including conclusions, the employed experimental designs, and the papers in which they are used so they can be easily looked up afterwards.

\subsubsection{The ExpML Experiment Markup Language}

Using the Exposé ontology as our core vocabulary, we can define a formal markup language for describing experiments, called ExpML. It is complementary to $\mathrm{PMML}^{5}$, which allows to exchange predictive models, but not detailed experimental setups nor evaluations. It is derived by translating the ontological classes and properties (relationships) to XML elements and syntax. This translation process is especially useful because it allows ontological extensions (e.g. to new machine learning tasks) to be translated into updated ExpML definitions.

Because ontological relationships are more expressive than XML syntax, different relationships between these concepts need to be translated quite differently. Table 1 provides a short overview of these relationships and their XML equivalent. Figure 2 illustrates this process, showing a real experiment (experiment 445080 in our experiment database) expressed in ExpML. The structure of this particular experiment is shown at the bottom of Fig. 3 . We assign an id to each operator and in- or output (e.g. datasets). For each operator, we state its inputs in an input data attribute, and for each output, we state the operator that generated that output in an output of attribute. As shown in the ExpML code, a dataset with $i d=$ ' $d 1$ ' is used as the input of workflow 'op1' and data processing operator 'op2'. The resulting dataset ' $\mathrm{d} 2$ ' is references as both the output of the operator 'op1' and workflow 'op2'.

Our data processing sub-workflow contains a single participant: a data processing application, i.e. a feature selection algorithm. It also requires an input, which will be the same dataset as before, and has a participant: an algorithm impl. It can also have multiple parameter settings and component settings, which will become XML subelements. Each component setting

5 See http://wWw.dmg. org/pmml-v3-2.html 

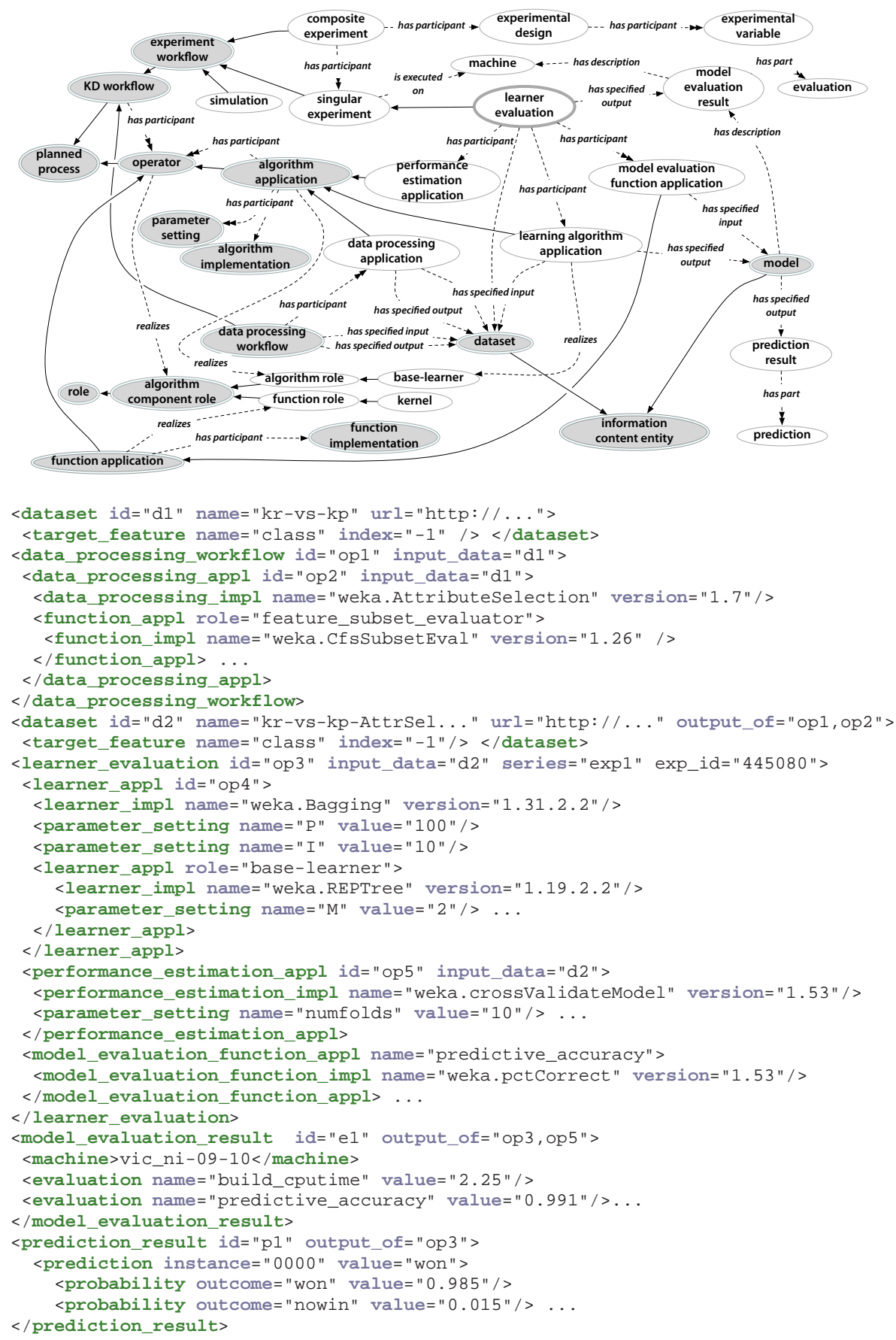

Fig. 2 Experimental workflows in Exposé (top) and ExpML (below). 


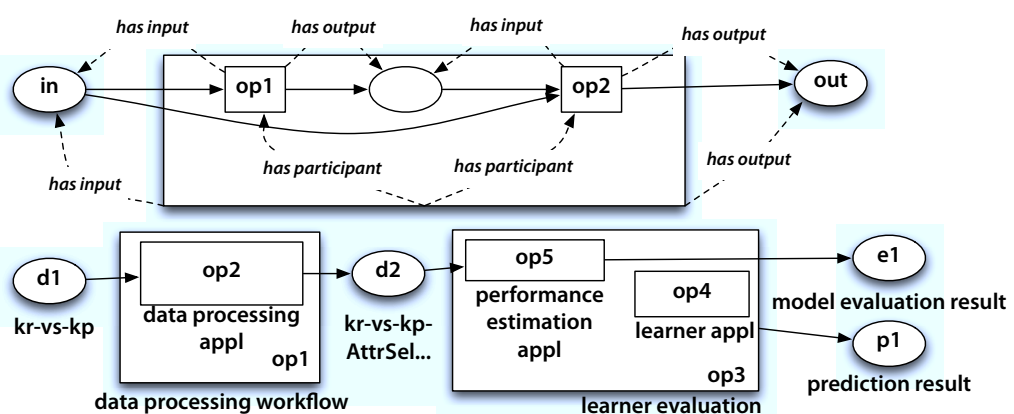

Fig. 3 Structure of a workflow (top) and an example (below, also see Fig. 2)

has a participant assumed to fulfill each of these roles. In the ontology, a realizes relationship indicates which processes can fulfill them. In the ExpML code, a function appl element is shows, with a role attribute signaling the role it is fulfilling.

In the second half of the workflow (see Figure 3), the generated dataset serves as the input for a learner evaluation, which will in turn produce a model evaluation result and a prediction result. The evaluation consists of a learning algorithm application complete with parameter and component settings (in this case including a base learner application with its own parameter settings), the performance estimation technique (10-fold cross-validation) and a list of evaluation functions to assess the produced models, each pointing to their precise implementations.

The output of the experiment is shown next, consisting of all evaluation results (also stating the machine used in order to interpret cpu time) and all predictions, including the probabilities for each class. Although omitted for brevity, evaluation error margins are stored as well. Storing predictions is especially useful if we want to apply new evaluation metrics afterwards without rerunning all prior experiments.

\subsubsection{The Experiment Databases (ExpDBs)}

Finally, all submitted ExpML descriptions are interpreted and automatically stored into (relational) databases. The database model, also based on Exposé, is very fine-grained, so that queries can be written about any aspect of the experimental setup, evaluation results, or properties of involved components (e.g., dataset size). A working implementation in MySQL, containing over 650,000 experiments, can be queried online. 


\subsubsection{Query Interfaces}

The database can be accessed through two query interfaces: an online interface on the homepage itself and an open-source desktop application. Both allow to launch queries written in SQL (many examples of SQL queries are supplied, including the ones used in Sect. 5), or composed in a graphical query interface, and can show the results in tables or graphical plots. The desktop application offers a wider range of plots, including self-organizing maps.

\subsection{Using the Database}

The three arrows emanating from the ExpDB at the bottom of Fig. 1 show different ways to tap into the stored information:

Querying This allows a researcher to formulate questions about the stored experiments, and immediately get all results of interest. Such queries could, for instance, be aimed at discovering ways in which an algorithm can be improved (e.g., see Sect. 5.2.1), after which that algorithm can be refined and tested again, thus completing the algorithm development cycle.

Mining A second use is to automatically look for patterns in algorithm performance by mining the stored results and theoretical meta-data. The insights provided by such meta-models can then be used to design better algorithms or to assist in knowledge discovery applications [8].

Integration Data mining toolboxes could also interface with ExpDBs directly, for instance to download the results of experiments that have been run before by a different user of that toolbox.

\subsection{Populating the Database}

The current database is populated with very diverse experiments to test algorithms under different conditions. First, we entered 54 classification algorithms from the WEKA platform together with all their parameters, 45 implementations of evaluation measures, 87 datasets from the UCI repository [3], 56 data characteristics calculated for each dataset, and two data preprocessors: correlation-based feature selection [14], and a subsampling procedure.

Next, three series of experiments were performed, in which a number of algorithms were explored more thoroughly than others:

- The first series simply ran all algorithms with default parameter settings.

- The second series varied each parameter, with at least 20 different values, of a selection of popular algorithms: SMO (a support vector machine (SVM) trainer), MultilayerPerceptron, J48 (C4.5), 1R, RandomForest, 
Bagging and Boosting. Moreover, different SVM kernels were used with their own parameter ranges, and all learners were used as base-learners for ensemble learners. We used a one-factor-at-a-time design to vary multiple parameters: each parameter (including the choice of base-learner or kernel) is varied in turn while keeping all others at default.

- Finally, the third series of experiments used a random sampling design to uniformly cover the entire parameter space (with at least 1000 settings) of an even smaller selection of algorithms: J48, Bagging and 1R.

All parameter settings were run on all datasets, and repeated 20 times with different random seeds for all algorithms that have them. In all cases, all 45 evaluation metrics were calculated in a 10-fold cross-validation procedure, with the same folds for each dataset. A large portion was additionally evaluated with a bias-variance analysis.

\subsubsection{Quality Control}

It is worth noting that, in the collaborative context, some form of quality control must be implemented to avoid contamination by bad (perhaps even fraudulent) ExpML descriptions. One solution, used in several repositories in bio-informatics, is to attach a trustworthiness value to the source of certain results. Experiments submitted from a trusted tool may be labeled very trustworthy, while custom submissions might get a lower value until the results are verified. Alternatively, if the database system can automatically run the algorithms in question, it could rerun all submitted experiments to verify the results.

\section{Learning from the Past}

In this section, we use the existing experiment database to illustrate how easily the results of previously stored experiments can be exploited for the discovery of new insights into a wide range of research questions, as well as to verify a number of recent studies. These illustrations can also be found in Vanschoren et al. [43]. Similar to Van Someren [37], we distinguish between three types of studies, increasingly making use of the available meta-level descriptions, and offering increasingly generalizable results:

1. Model-level analysis. These studies evaluate the produced models through a range of performance measures, but typically consider only individual datasets and algorithms. They typically try to identify HOW a specific algorithm performs, either on average or under specific conditions.

2. Data-level analysis. These studies investigate how known or measured data properties, not individual datasets, affect the performance of specific algo- 
rithms. They identify WHEN (on which kinds of data) an algorithm can be expected to behave a certain way.

3. Method-level analysis. These studies don't look at individual algorithms, but take general properties of the algorithms (eg. their bias-variance profile) into account to identify WHY an algorithm behaves a certain way.

\subsection{Model-level Analysis}

In the first type of study, we are interested in how individual algorithms perform on specific datasets. This type of study is typically used to benchmark, compare or rank algorithms, but also to investigate how specific parameter settings affect performance.

\subsubsection{Comparing Algorithms}

To compare the performance of all algorithms on one specific dataset, we write a query that simply selects the name of the algorithm used and the predictive accuracy recorded in all stored experiments on, for instance, the dataset 'letter'. A graph representation of this query is shown in Fig. 4. It joins the tables (nodes in the graph) of the learning algorithm, dataset, and evaluation based on the experiment in which they are used. It also selects the algorithm name and its evaluation (in brackets), and adds constraints (in ellipses) on the dataset name and the evaluation metric used. is_original indicates that the dataset is not preprocessed. For more detail, we can also select the kernel in the case of a SVM and the base-learner in the case of an ensemble. This is done in the subqueries shown in the bottom of Fig. 4. We order the results by their performance and plot the results in Fig. 5 .

Since the returned results are always as general as the query allows, we now have a complete overview of how each algorithm performed. Next to their optimal performance, it is also immediately clear how much variance is caused by suboptimal parameter settings (at least for those algorithms whose parameters were varied). For instance, when looking at SVMs, it is clear that especially the RBF-kernel is of great use here (indeed, RBF kernels are popular in letter recognition problems), while the polynomial kernel is much less interesting. However, there is still much variation in the performance of the SVM's, so it might be interesting to investigate this in more detail. Also, while most algorithms vary smoothly as their parameters are altered, there seem to be large jumps in the performances of SVMs and RandomForests, which are, in all likelihood, caused by parameters that heavily affect their performance. Moreover, when looking at bagging and boosting, it is clear that some base-learners are much more interesting than others. For instance, it appears that while bagging and boosting do give an extra edge to the nearest 


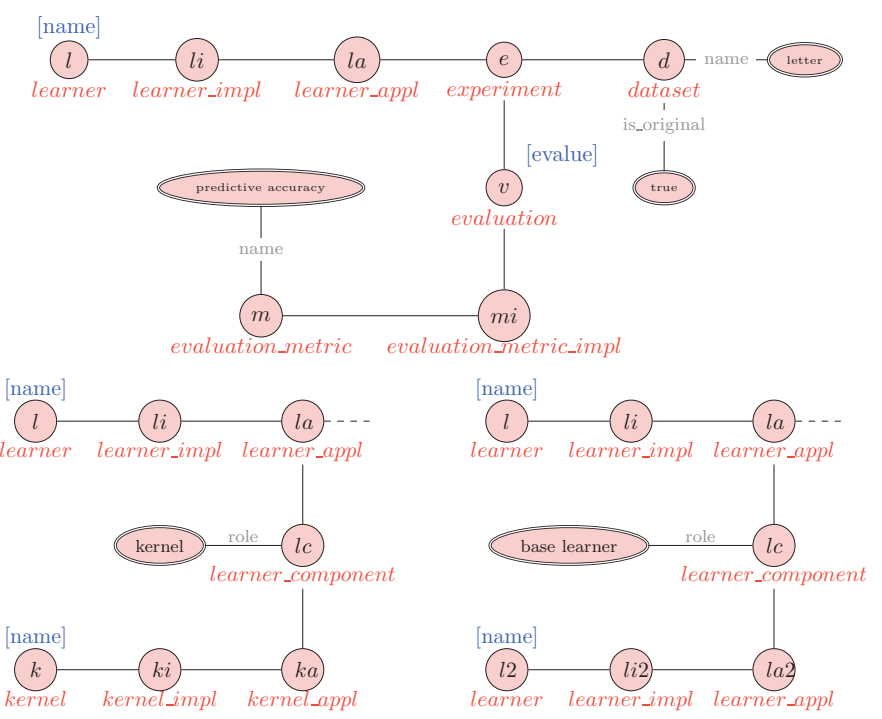

Fig. 4 A graph representation of our query. The top shows the main query, and below are two subqueries selecting the used kernels (left) and the base-learners of an ensemble method (right).

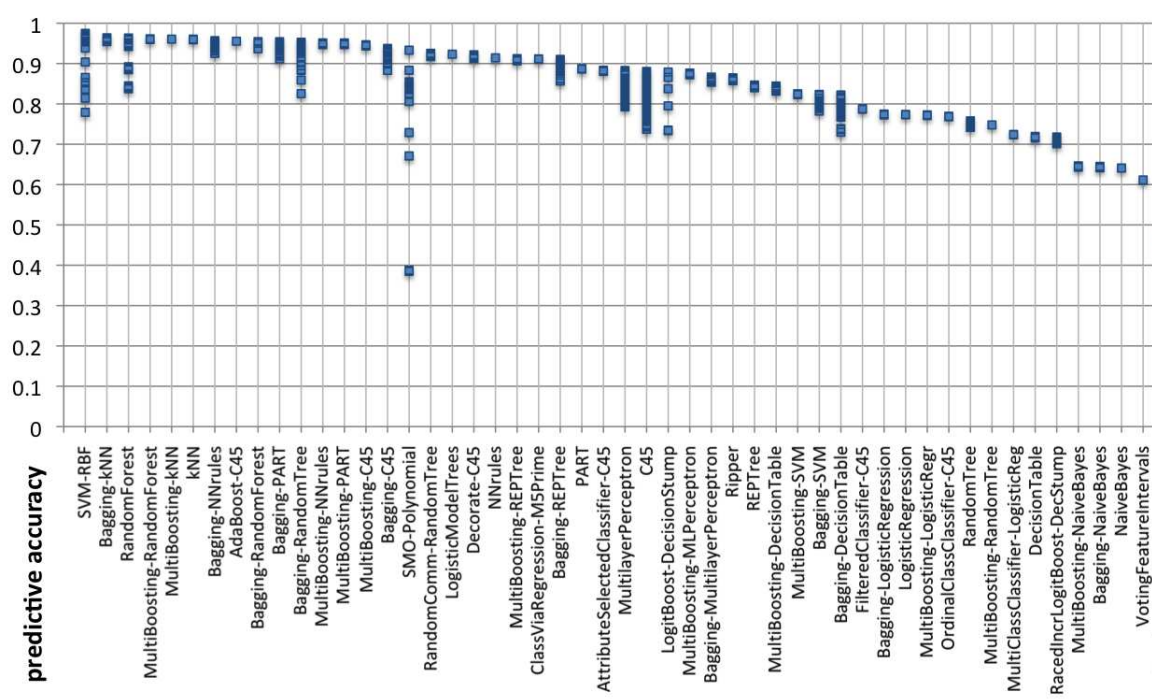

Fig. 5 Performance of algorithms on dataset 'letter'.

neighbor and logistic regression algorithms, the effect is rather limited. Conversely, bagging RandomTree seems to be hugely profitable, but this does not hold for boosting. It also seems more rewarding to fine-tune RandomForests, MultiLayerPerceptrons and SVMs than to bag or boost their default setting. 

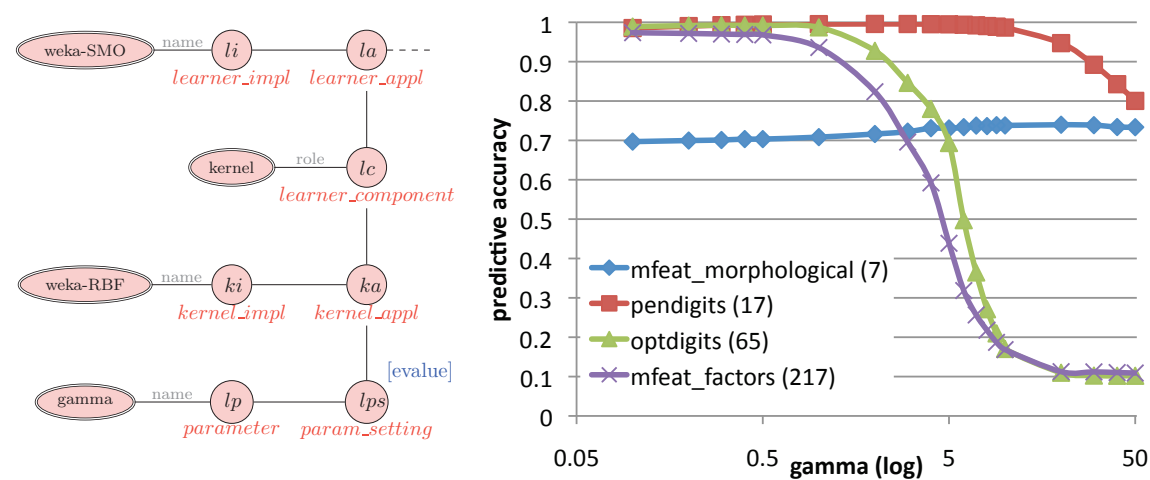

Fig. 6 The effect of parameter gamma of the RBF-kernel in SVMs on a number of different datasets, with their number of attributes shown in parentheses, and the accompanying query graph.

Still, this is only one dataset, further querying is needed. Given the generality of the results, each query is likely to highlight things we were not expecting, providing interesting cases for further study.

\subsubsection{Investigating Parameter Effects}

First, we examine the effect of the parameters of the RBF kernel. Based on the first query, we can focus on the SVM's results by adding a constraint. Then we simply ask for the value of the parameter we are interested in. By selecting the value of the gamma parameter and plotting the results, we obtain Fig. 6 . We constrain the datasets to a selection with the same default accuracy $(10 \%)$.

On the 'mfeat_morphological' (and 'pendigits') dataset, performance increases when increasing gamma up to a point, after which it slowly declines. The other curves show that the effect on accuracy on other datasets is very different: performance is high for low gamma values, but quickly drops down to the default accuracy for higher values. Looking at the number of attributes in each dataset (shown in parentheses) we can observe some correlation.

A possible explanation for this lies in the fact that this SVM implementation normalizes all attributes into the interval $[0,1]$. Therefore, the maximal squared distance between two examples, $\sum\left(a_{i}-b_{i}\right)^{2}$ for every attribute $i$, is equal to the number of attributes. Since the RBF-kernel computes $e^{\left(-\gamma * \sum\left(a_{i}-b_{i}\right)^{2}\right)}$, the kernel value will go to zero very quickly for large gammavalues and a large number of attributes, making the non-zero neighborhood around a support vector very small. Consequently, the SVM will overfit these support vectors, resulting in low accuracies. This suggests that the RBF kernel should take the number of attributes into account to make the default 


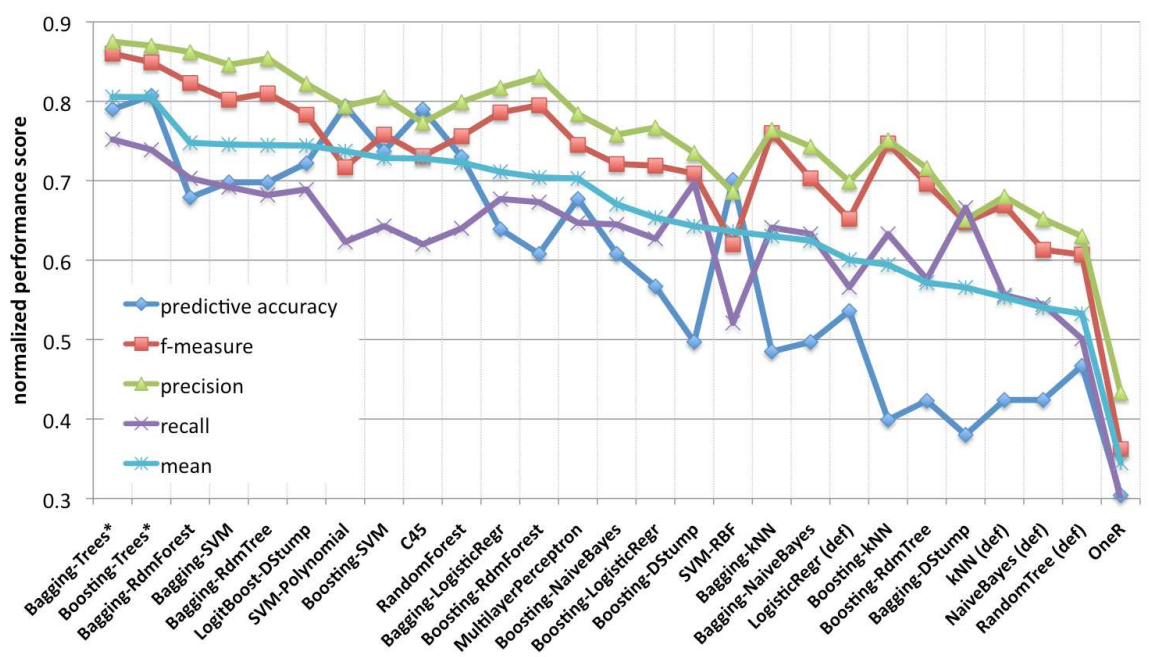

Fig. 7 Ranking of algorithms over all datasets on different performance metrics.

gamma value more suitable across a range of datasets. It also illustrates how the experiment database allows the investigation of algorithms in detail and assist their development.

\subsubsection{General Comparisons}

By simply dropping the constraints on the datasets used, the query will return the results over a large number of different problems. Furthermore, to compare algorithms over a range of performance metrics, instead of only considering predictive accuracy, we can use a normalization technique used by Caruana and Niculescu-Mizil [11]: normalize all performance metrics between the baseline performance and the best observed performance over all algorithms on each dataset. Using the aggregation functions of SQL, we can do this normalization on the fly, as part of the query.

We can now perform a very general comparison of supervised learning algorithms. We select all algorithms whose parameters were varied (see Sect. 4.3) and, though only as a point of comparison, logistic regression, nearest neighbors (kNN), naive Bayes and RandomTree with their default parameter settings. As for the performance metrics, we selected predictive accuracy, F-measure, precision and recall, the last three of which were averaged over all classes. We then queried for the maximal (normalized) performance of each algorithm for each metric on each dataset, averaged each of these scores 
over all datasets, and finally ranked all classifiers by the average of predictive accuracy, precision and recall. ${ }^{6}$ The results of this query are shown in Fig. 7.

The overall best performing algorithms are mostly bagged and boosted ensembles. Especially bagged and boosted trees perform very well, in agreement with the previous results [11]. In Fig. 7 these are grouped as Trees* since they perform very similarly, and include C4.5, PART, Ripper, NaiveBayesTree, REPTree and similar tree-based learners. Another shared conclusion is that boosting full trees performs dramatically better than boosting stumps (see Boosting-DStump) or boosting random trees. While C45 seems to perform slightly better than RandomForests on predictive accuracy, this is only the case for multi-class datasets. When constraining the results to binary datasets (not shown here), RandomForests do outperform C45 on all metrics.

Furthermore, since this study contains many more algorithms, we can make a number of additional observations. For instance, the bagged versions of most strong learners (SVM, C45, RandomForest, etc.) seem to improve primarily on precision and recall, while the original base-learners (with optimized parameters) perform better on predictive accuracy. Apparently, tuning the parameters of these strong learners has a much larger effect on accuracy than on the other metrics, for which it is better to employ bagging than parameter tuning, at least on multi-class datasets.

\subsection{Data-level Analysis}

While the queries in the previous section allow the examination of the behavior of learning algorithms to a high degree of detail, they give no indication of exactly when (on which kind of datasets) certain behavior is to be expected. In order to obtain results that generalize over different datasets, we need to look at the properties of each individual dataset, and investigate how they affect learning performance.

\subsubsection{Data Property Effects}

In a first such study, we examine what causes the 'performance jumps' that we noticed with the RandomForest algorithm in Fig. 5. Querying for the effects of the number of trees in the forest and the dataset size yields Fig. 8.

\footnotetext{
6 Since all algorithms were evaluated over all of the datasets (with 10-fold crossvalidation), we could not optimize their parameters on a separate calibration set for this comparison. To limit the effect of overfitting, we only included a limited set of parameter settings, all of which fairly close to the default setting. Nevertheless, these results should be interpreted with caution as they might be overly optimistic.
} 


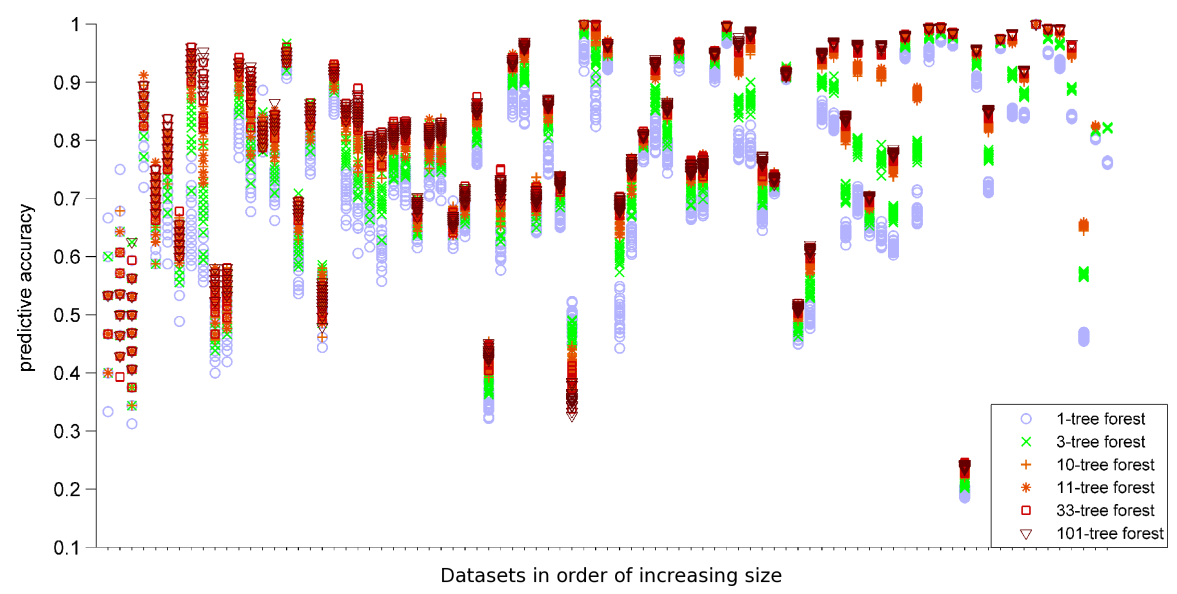

Fig. 8 The effect of dataset size and the number of trees for random forests. The dataset names are omitted since they are too small to be printed legibly.

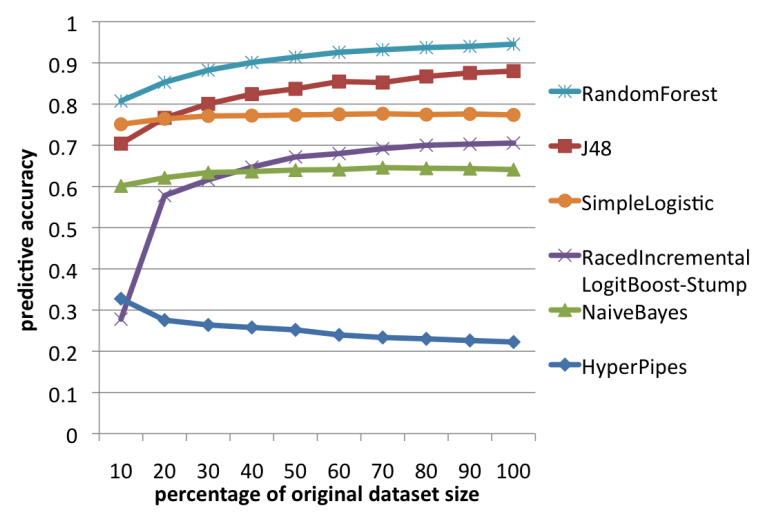

Fig. 9 Learning curves on the Letter-dataset.

This shows that predictive accuracy increases with the number of trees, usually leveling off between 33 and 101 trees. ${ }^{7}$ One dataset, monks problems 2 , is a notable exception: obtaining less than $50 \%$ accuracy on a binary problem, it actually performs worse as more trees are included. We also see that on large datasets, the accuracies for a given forest size vary less since the trees become more stable on large datasets, thus causing clear performance jumps on very large datasets. However, for very small datasets, the benefit of using more trees is overpowered by the randomization occurring in the trees (the algorithm considers $\mathrm{K}$ random features at each node).

7 We used a geometric progression $(1,3,11,33,101)$ of the number of trees, choosing for odd numbers to break ties while voting. 


\subsubsection{Preprocessing Effects}

We can also study the effect of preprocessing methods. For instance, to investigate if the results in Fig. 2 are also valid on smaller samples, we can query for the results on downsampled versions of the dataset, yielding a learning curve for each algorithm, as shown in Fig. 9. This provides further evidence that the ranking of algorithms depends on the size of the dataset sample [30]. While logistic regression is initially stronger than J48, the latter keeps on improving when given more data. Also note that RacedIncrementalLogitBoost has a particularly steep learning curve, crossing two other curves, and that the performance of the HyperPipes algorithm actually worsens given more data, which suggests it was 'lucky' on the smaller samples.

\subsubsection{Mining for Patterns in Learning Behavior}

Instead of studying different dataset properties independently, we could also use data mining techniques to relate the effect of many different properties to an algorithm's performance. For instance, when looking at Fig. 7, we see that OneR performs obviously much worse than the other algorithms. Still, some earlier studies, most notably one by Holte [18], found very little performance differences between OneR and the more complex J48. To study this discrepancy in more detail, we can query for the default performance of OneR and J48 on all UCI datasets, and plot them against each other, as shown in Fig. 10(a). This shows that on some datasets, the performances are similar (crossing near the diagonal), while on others, J48 is the clear winner. Discretizing these results into three classes as shown in Fig. 10(a), and querying for the characteristics of each dataset, we can train a meta-decision tree predicting on which kinds of datasets J48 has the advantage (see Fig. 10(b)). From this we learn that a high number of class values often leads to a large win of J48 over OneR. Indeed, the original study [18] only had one dataset with that many classes.

\subsection{Method level analysis}

While the results in the previous section are clearly more generalizable towards the datasets used, they don't explain why algorithms behave a certain way. They only consider individual algorithms and thus do not generalize over different techniques. Hence, we need to include algorithm properties in our queries as well. 


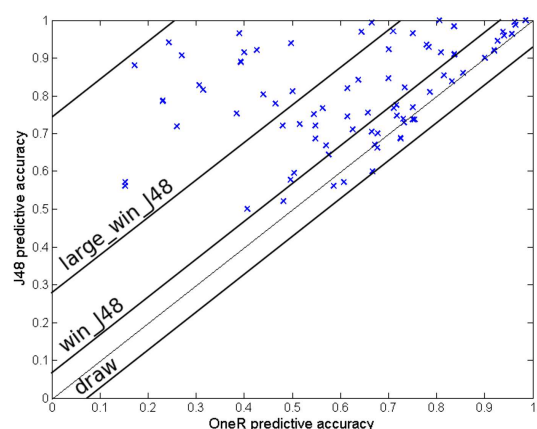

(a)

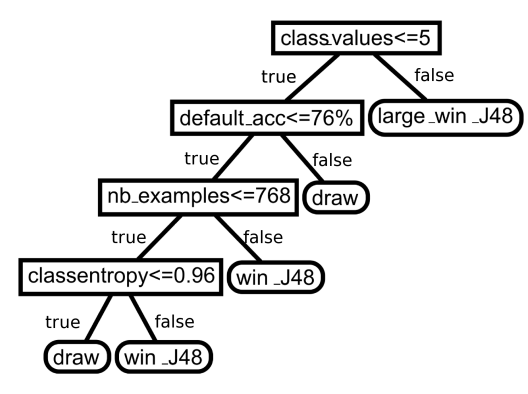

(b)

Fig. 10 (a) J48's performance against OneR's for all datasets, discretized into 3 classes. (b) A meta-decision tree predicting algorithm superiority based on data characteristics.

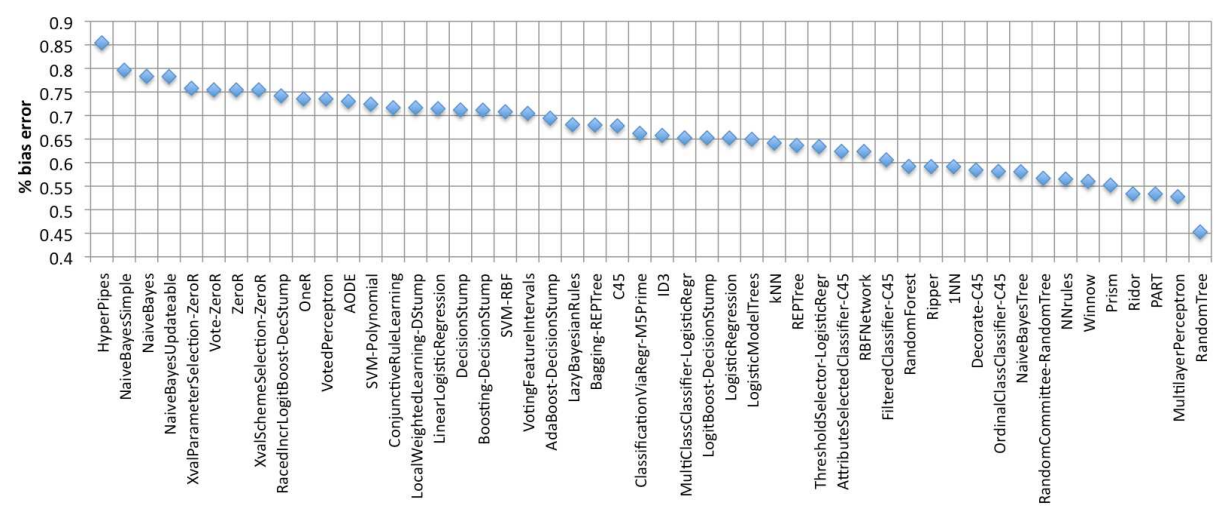

Fig. 11 Average percentage of bias-related error for each algorithm over all datasets.

\subsubsection{Bias-variance Profiles}

One very interesting property of an algorithm is its bias-variance profile [20]. Since the database contains a large number of bias-variance decomposition experiments, we can give a realistic, numerical assessment of how capable each algorithm is in reducing bias and variance error. In Fig. 11 we show, for each algorithm, the proportion of the total error that can be attributed to bias error, using default parameter settings and averaged over all datasets.

The algorithms are ordered from large bias (low variance), to low bias (high variance). NaiveBayes is, as expected, one of the algorithms with the strongest variance management (it avoids overfitting), but poor bias management (the ability to model complex target concepts). RandomTree, on the other hand, has very good bias management, but generates more variance error. When 


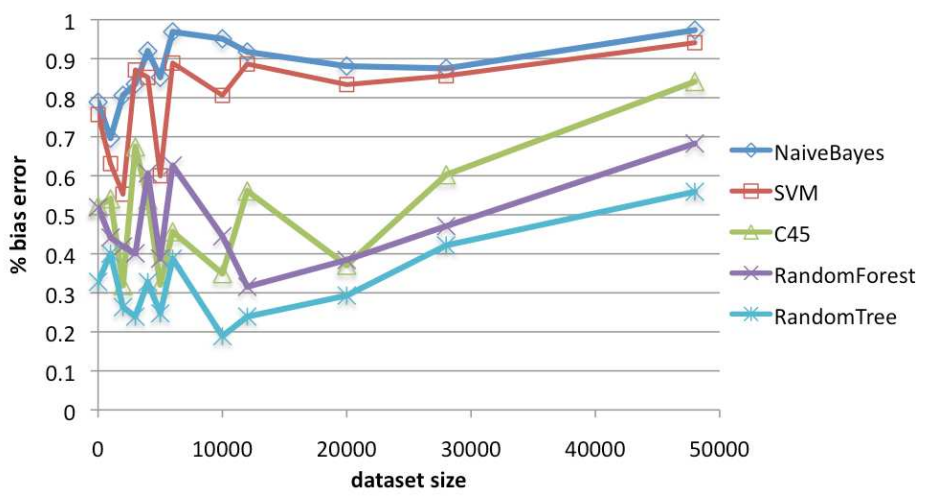

Fig. 12 Average percentage of bias-related error in algorithms vs dataset size.

looking at ensemble methods, it shows that bagging reduces variance, as it causes REPTree to shift significantly to the left. Conversely, boosting reduces bias, shifting DecisionStump to the right in AdaBoost and LogitBoost.

\subsubsection{Bias-variance Effects}

As a final study, we investigate the claim by Brain and Webb [7] that on large datasets, the bias-component of the error becomes the most important factor, and that we should use algorithms with high bias management to tackle them. To verify this, we look for a connection between the dataset size and the proportion of bias error in the total error of a number of algorithms, using the previous figure to select algorithms with very different bias-variance profiles. By plotting the percentage of bias error generated on each dataset against the size of that dataset we obtain Fig. 12. Datasets or similar size are grouped for legibility. It shows that bias error is of varying significance on small datasets, but steadily increases in importance on larger datasets, for all algorithms. This validates the previous study on a much larger set of datasets. In this case (on UCI datasets), bias becomes the most important factor on datasets larger than 50000 examples, no matter which algorithm is used. As such, it is indeed advisable to look to algorithms with good bias management when dealing with large datasets, as variance becomes a less important factor. 


\section{Conclusions}

Experiment databases are databases specifically designed to collect all the details on large numbers of past experiments, possibly performed by many different researchers, and make them immediately available to everyone. They ensure that experiments are repeatable and automatically organize them so that they can be easily reused in future studies.

In this chapter, we have provided a high-level overview of their design. Similar to experiment repositories actively used in e-Sciences, it consists of an ontological domain model, which is in turn used to create a formal experiment description language and a detailed database model.

We also used an existing experiment database to illustrate how easily the results of previously stored experiments can be exploited to gain new insight: we performed elaborate algorithms comparisons, investigated the effects of algorithm parameters and data properties, suggested algorithm improvements, built meta-models of algorithm performance, and studied the bias-variance profiles of learning algorithms, each time by writing only a single query.

Experiment databases offer the possibility to truly unite the results of many individual machine learning studies, enhance cooperation, and facilitate large-scale empirical studies. As such, we are confident that they can contribute greatly to the vigor of machine learning research.

Our database is available online at http://expdb.cs.kuleuven.be

\section{Acknowledgements}

Hendrik Blockeel was a Postdoctoral Fellow of the Fund for Scientific Research - Flanders (Belgium) (F.W.O.-Vlaanderen) at the time of this work, and this research is further supported by GOA 2003/08 "Inductive Knowledge Bases" and F.W.O.-Vlaanderen G.0108.06 "Foundations of Inductive Databases for Data Mining".

\section{References}

1. Aha, D.: Generalizing from case studies: A case study. Proceedings of the Ninth International Conference on Machine Learning pp. 1-10 (1992)

2. Ashburner, M., Ball, C.A., Blake, J.A., Botstein, D., Butler, H., Cherry, J.M., Davis, A.P., Dolinski, K., Dwight, S.S., Eppig, J.T., Harris, M.A., Hill, D.P., IsselTarver, L., Kasarskis, A., Lewis, S., Matese, J.C., Richardson, J.E., Ringwald, M., Rubin, G.M., Sherlock, G.: Gene ontology: tool for the unification of biology. nature genetics 25, 25-29 (2000)

3. Asuncion, A., Newman, D.: UCI machine learning repository. University of California, School of Information and Computer Science (2007) 
4. Ball, C., Brazma, A., Causton, H., Chervitz, S.: Submission of microarray data to public repositories. PLoS Biology 2(9), e317 (2004)

5. Blockeel, H.: Experiment databases: A novel methodology for experimental research. Lecture Notes in Computer Science 3933, 72-85 (2006)

6. Blockeel, H., Vanschoren, J.: Experiment databases: Towards an improved experimental methodology in machine learning. Lecture Notes in Computer Science 4702, 6-17 (2007)

7. Brain, D., Webb, G.: The need for low bias algorithms in classification learning from large data sets. PKDD '02: Proceedings of the 6th European Conference on Principles of Data Mining and Knowledge Discovery pp. 62-73 (2002)

8. Brazdil, P., Giraud-Carrier, C., Soares, C., Vilalta, R.: Metalearning: Applications to data mining. Springer (2009)

9. Brazma, A., Hingamp, P., Quackenbush, J., Sherlock, G., Spellman, P., Stoeckert, C., Aach, J., Ansorge, W., Ball, C.A., Causton, H.C., Gaasterland, T., Glenisson, P., Holstege, F.C., Kim, I.F., Markowitz, V., Matese, J.C., Parkinson, H., Robinson, A., Sarkans, U., Schulze-Kremer, S., Stewart, J., Taylor, R., Vingron, J.V.M.: Minimum information about a microarray experiment. nature genetics 29, $365-371(2001)$

10. Brown, D., Vogt, R., Beck, B., Pruet, J.: High energy nuclear database: a testbed for nuclear data information technology. International Conference on Nuclear Data for Science and Technology p. Article 250 (2007)

11. Caruana, R., Niculescu-Mizil, A.: An empirical comparison of supervised learning algorithms. Proceedings of the 23rd International Conference on Machine Learning (ICML'06) pp. 161-168 (2006)

12. Chandrasekaran, B., Josephson, J.: What are ontologies, and why do we need them? IEEE Intelligent systems 14(1), 20-26 (1999)

13. Derriere, S., Preite-Martinez, A., Richard, A.: UCDs and ontologies. ASP Conference Series 351, 449 (2006)

14. Hall, M.: Correlation-based feature selection for machine learning. Ph.D dissertation Hamilton, NZ: Waikato University, Department of Computer Science (1998)

15. Hall, M., Frank, E., Holmes, G., Pfahringer, B., Reutemann, P., Witten, I.: The weka data mining software: An update. SIGKDD Explorations 11(1), 10-18 (2009)

16. Hilario, M., Kalousis, A., Nguyen, P., Woznica, A.: A data mining ontology for algorithm selection and meta-mining. Proceedings of the ECML/PKDD09 Workshop on 3rd generation Data Mining (SoKD-09) pp. 76-87 (2009)

17. Hirsh, H.: Data mining research: Current status and future opportunities. Statistical Analysis and Data Mining 1(2), 104-107 (2008)

18. Holte, R.: Very simple classification rules perform well on most commonly used datasets. Machine Learning 11, 63-91 (1993)

19. Hoste, V., Daelemans, W.: Comparing learning approaches to coreference resolution. there is more to it than bias. Proceedings of the Workshop on Meta-Learning (ICML-2005) pp. 20-27 (2005)

20. Kalousis, A., Hilario, M.: Building algorithm profiles for prior model selection in knowledge discovery systems. Engineering Intelligent Systems 8(2) (2000)

21. Keogh, E., Kasetty, S.: On the need for time series data mining benchmarks: A survey and empirical demonstration. Data Mining and Knowledge Discovery 7(4), 349-371 (2003)

22. Kietz, J., Serban, F., Bernstein, A., Fischer, S.: Towards cooperative planning of data mining workflows. Proceedings of the Third Generation Data Mining Workshop at the 2009 European Conference on Machine Learning (ECML 2009) pp. 1-12 (2009) 
23. King, R., Rowland, J., Oliver, S., Young, M., Aubrey, W., Byrne, E., Liakata, M., Markham, M., Pir, P., Soldatova, L., Sparkes, A., Whelan, K., Clare, A.: The automation of science. Science 324(3)(5923), 85-89 (2009)

24. Manolescu, I., Afanasiev, L., Arion, A., Dittrich, J., Manegold, S., Polyzotis, N., Schnaitter, K., Senellart, P., Zoupanos, S.: The repeatability experiment of SIGMOD 2008. ACM SIGMOD Record 37(1) (2008)

25. Michie, D., Spiegelhalter, D., Taylor, C.: Machine learning, neural and statistical classification. Ellis Horwood (1994)

26. Nielsen, M.: The future of science: Building a better collective memory. APS Physics 17(10) (2008)

27. Ochsenbein, F., Williams, R., Davenhall, C., Durand, D., Fernique, P., Hanisch, R., Giaretta, D., McGlynn, T., Szalay, A., Wicenec, A.: Votable: tabular data for the virtual observatory. Toward an International Virtual Observatory. Springer pp. 118-123 (2004)

28. Panov, P., Soldatova, L., Džeroski, S.: Towards an ontology of data mining investigations. Discovery Science (DS09). Lecture Notes in Artificial Intelligence 5808, 257-271 (2009)

29. Pedersen, T.: Empiricism is not a matter of faith. Computational Linguistics 34, 465-470 (2008)

30. Perlich, C., Provost, F., Simonoff, J.: Tree induction vs. logistic regression: A learning-curve analysis. The Journal of Machine Learning Research 4, 211-255 (2003)

31. Pfahringer, B., Bensusan, H., Giraud-Carrier, C.: Meta-learning by landmarking various learning algorithms. Proceedings of the Seventeenth International Conference on Machine Learning pp. 743-750 (2000)

32. Schaaff, A.: Data in astronomy: From the pipeline to the virtual observatory. Lecture Notes in Computer Science 4832, 52-62 (2007)

33. Soldatova, L., King, R.: An ontology of scientific experiments. Journal of the Royal Society Interface 3(11), 795-803 (2006)

34. Sonnenburg, S., Braun, M., Ong, C., Bengio, S., Bottou, L., Holmes, G., LeCun, Y., Muller, K., Pereira, F., Rasmussen, C.E., Ratsch, G., Scholkopf, B., Smola, A., Vincent, P., Weston, J., Williamson, R.: The need for open source software in machine learning. Journal of Machine Learning Research 8, 2443-2466 (2007)

35. Stoeckert, C., Causton, H., Ball, C.: Microarray databases: standards and ontologies. nature genetics 32, 469-473 (2002)

36. Szalay, A., Gray, J.: The world-wide telescope. Science 293, 2037-2040 (2001)

37. Van Someren, M.: Model class selection and construction: Beyond the procrustean approach to machine learning applications. Lecture Notes in Computer Science 2049, 196-217 (2001)

38. Vanschoren, J., Van Assche, A., Vens, C., Blockeel, H.: Meta-learning from experiment databases: An illustration. Proceedings of the 16th Annual Machine Learning Conference of Belgium and The Netherlands (Benelearn07) pp. 120$127(2007)$

39. Vanschoren, J., Blockeel, H.: Investigating classifier learning behavior with experiment databases. Data Analysis, Machine Learning and Applications: 31st Annual Conference of the Gesellschaft für Klassifikation pp. 421-428 (2008)

40. Vanschoren, J., Blockeel, H.: A community-based platform for machine learning experimentation. Lecture Notes in Artificial Intelligence 5782, 750-754 (2009)

41. Vanschoren, J., Blockeel, H., Pfahringer, B.: Experiment databases: Creating a new platform for meta-learning research. Proceedings of the ICML/UAI/COLT Joint Planning to Learn Workshop (PlanLearn08) pp. 10-15 (2008)

42. Vanschoren, J., Blockeel, H., Pfahringer, B., Holmes, G.: Organizing the world's machine learning information. Communications in Computer and Information Science 17, 693-708 (2008) 
43. Vanschoren, J., Pfahringer, B., Holmes, G.: Learning from the past with experiment databases. Lecture Notes in Artificial Intelligence 5351, 485-492 (2008)

44. Vizcaino, J.A., Cote, R., Reisinger, F., Foster, J.M., Mueller, M., Rameseder, J., Hermjakob, H., Martens, L.: A guide to the proteomics identifications database proteomics data repository. Proteomics 9(18), 4276-4283 (2009)

45. Yasuda, N., Mizumoto, Y., Ohishi, M., amd T Budavári, W.O., Haridas, V., Li, N., Malik, T., Szalay, A., Hill, M., Linde, T., Mann, B., Page, C.: Astronomical data query language: Simple query protocol for the virtual observatory. ASP Conference Proceedings 314, 293 (2004)

46. Žáková, M., Kremen, P., Železný, F., Lavrač, N.: Planning to learn with a knowledge discovery ontology. Second planning to learn workshop at the joint ICML/COLT/UAI Conference pp. 29-34 (2008) 